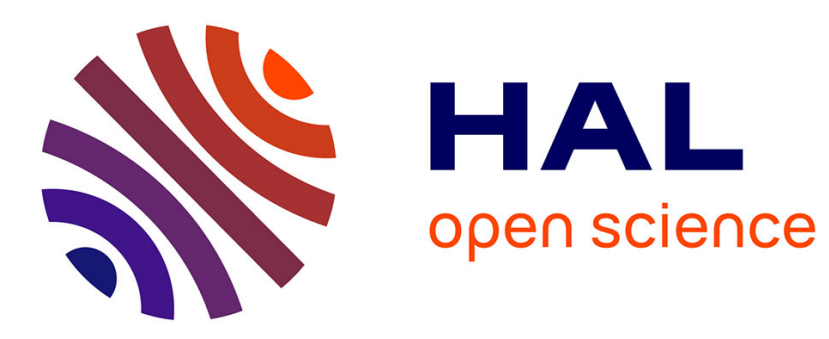

\title{
Contactless thin-film rheology unveiled by laser-induced nanoscale interface dynamics
}

\author{
Gopal Verma, Hugo Chesneau, Hamza Chraibi, Ulysse Delabre, Regis \\ Wunenburger, Jean-Pierre Delville
}

\section{- To cite this version:}

Gopal Verma, Hugo Chesneau, Hamza Chraibi, Ulysse Delabre, Regis Wunenburger, et al.. Contactless thin-film rheology unveiled by laser-induced nanoscale interface dynamics. Soft Matter, 2020, 10.1039/D0SM00978D . hal-02915823

\section{HAL Id: hal-02915823 \\ https://hal.science/hal-02915823}

Submitted on 16 Aug 2020

HAL is a multi-disciplinary open access archive for the deposit and dissemination of scientific research documents, whether they are published or not. The documents may come from teaching and research institutions in France or abroad, or from public or private research centers.
L'archive ouverte pluridisciplinaire HAL, est destinée au dépôt et à la diffusion de documents scientifiques de niveau recherche, publiés ou non, émanant des établissements d'enseignement et de recherche français ou étrangers, des laboratoires publics ou privés. 


\title{
Soft Matter
}

\section{ARTICLE}

\section{Contactless thin-film rheology unveiled by laser-induced nanoscale interface dynamics}

Received 00th January 20xx Accepted 00th January 20xx

DOI: $10.1039 / \times 0 \times x 00000 x$

www.rsc.org/

\author{
Gopal Verma, ${ }^{* a}$ Hugo Chesneau, ${ }^{a}$ Hamza Chraïbi, ${ }^{a}$ Ulysse Delabre, ${ }^{a}$ Régis Wunenburger ${ }^{b}$ and Jean- \\ Pierre Delville, ${ }^{* a}$
}

\begin{abstract}
One of the classical limitations for the investigation of the local rheology of small scale soft objects and/or confined fluids is related to the difficulty to control mechanical contact and its consequences. In order to overcome these issues, we implement a new local, active, fast and contactless optical strategy, called optorheology, which is based on both the optical radiation pressure of a laser wave to dynamically deform a fluid interface and interferometry to probe this deformation with nanometric resolution. This optical approach is first validated by measuring the surface tension and the viscosity of transparent Newtonian liquids. We also show how non-equilibrium situations, such as continuous evaporation, can be used to deduce the thickness dependence of the rheology of thin films and the concentration dependence of the viscosity of binary liquid mixtures and suspensions. We further extend the investigation to elasticity and viscoelasticity measurements of polymer solutions. Finally, since liquids may absorb light, we discuss the influence of a weak laser heating and the triggering of interface deformations by thermocapillary tangential stresses that could represent a complementary approach to probe the rheology at small scale.
\end{abstract}

\section{Introduction}

The last decades have seen a tremendous development of thin liquid film technologies for lubrication, ${ }^{1}$ advanced coating ${ }^{2}$ or micro-/nano-lithography. ${ }^{3}$ Due to their importance in many areas, $4,5,6$ these technologies started to integrate small scale complexity and functionalities for surface chemistry, biological transfer, sensing or photonics applications using spin or dipcoated liquid mixtures, ${ }^{7}$ suspensions $^{8}$ or buffer solutions embedding cells. ${ }^{9}$ The control of thin film and drop evaporation is also advanced for innovating painting, surface patterning ${ }^{10}$ or organic electronics ${ }^{11}$ after drying. These new applications of thin films then require a perfect knowledge of the film or drop properties in real time as (i) the dynamics may vary with the height at small scale, ${ }^{12}, 13$ and (ii) the rheology and interfacial properties may nonlinearly depend on the solute concentration, particularly during evaporation where all these properties become time-dependent. However, the characterization of the rheology when at least one dimension (the height of a film or of a sessile radius) reaches the microscale becomes difficult because many methods, even miniaturized, utilize contacts (of a sphere or a tip for example) that make them invasive. They may also be hard to implement in situ due to confinement, and their dynamics is often too slow, a clear drawback for evolving systems such as evaporating liquid mixtures.

a. Univ. Bordeaux, LOMA, CNRS, UMR 5798, 33405 Talence, France.

E-mail : gverma05@gmail.com; jean-pierre.delville@u-bordeaux.fr

b. Sorbonne Université, CNRS, Institut Jean Le Rond d'Alembert, F-75005 Paris,

France
These requirements prompted the emergence of contactless micro-rheology techniques. Among all the various methods advanced, those based on the analysis of interface deformation have attracted attention for several important reasons: (i) most of the targeted properties of the liquid can be deduced from the analysis of both the dynamical and stationary deformations of the interface in different regimes (inertial, overdamped, ...); (ii) Interfacial deformations can be triggered without any contact using several ways: from natural thermal fluctuations, ${ }^{14}$ by capillary levelling, ${ }^{13,15}$ by applying an electrostatic stress, ${ }^{16}, 17,18$ by triggering tangential thermal gradient and thermocapillary stresses, $19,20,21$ or by irradiating the interface using the radiation pressure of an acoustic $22,23,24$ or a laser beam. ${ }^{25},{ }^{26}$ The fluctuation method is based on the analysis of light scattering by broadband thermal fluctuations at the free surface and then it requires long signal acquisition durations. Electric actuation is much faster, but extremely sensitive to sample vibrations, which can easily produce irreversible contact between the sample and the tip-like electrodes due to the close distance separating them. Thermocapillary stresses require well-controlled thermal gradients that strongly depend on boundary conditions. The optical and acoustic deformation techniques thus appear as the most adapted ones for in situ contactless measurements since they do not require dedicated fabrication except the presence of a small optical/acoustic pathway for the wave interaction. Both techniques have drawbacks and advantages: (i) wave absorption and the associated heating may be an issue, (ii) since the sound velocity is much smaller than the light celerity, acoustic radiation pressure is intrinsically much more intense than the optical one; (iii) as optical wavelengths are much smaller than acoustic ones at usual ultrasonic frequencies, optical beams are much more focusable than 
acoustic ones and allow to probe surfaces of much smaller extent. We thus choose the optical route to actuate liquid interfaces and measure rheological properties without any contact.

Since the seminal work of Ashkin \& Dziedzic on the Abraham-Minkhowski controversy, ${ }^{27}$ the radiation pressure of laser waves has been used to deform liquid interfaces $28,29,30$ and these deformations were foreseen later on as a way to characterize the involved liquids. Nonetheless, the optical radiation pressure exerted on a free surface at normal incidence $\Pi_{\text {rad }} \approx(n-1) I_{0} / c$ remains weak; $n$ is the refractive index of the liquid and $I_{0}=2 P /\left(\pi \omega_{0}^{2}\right)$ is the beam-axis intensity of the incident laser beam, where $P$ and $\omega_{0}$ are respectively the power and the waist of the Gaussian wave. For $(n-1) \simeq 0.4$ typical of liquid-air interfaces and mean values $P=1 \mathrm{~W}$ and $\omega_{0}=100 \mu \mathrm{m}$ for continuous laser beams, the radiation pressure is on the order of a tenth of Pascals. The stationary height $h$ of the resulting interface deformation of a film is deduced from the balance between the radiation pressure $\Pi_{\text {rad }}$, and both the Laplace pressure $\Pi_{\text {Lap }}=\gamma \kappa$ and hydrostatic pressure $\rho g h ; \gamma$ is the surface tension, $\kappa \approx h / \omega_{0}^{2}$ the interface curvature on beam axis and $\rho$ the liquid density. Note that the hydrostatic pressure effect is often neglected at small scale since the optical Bond number $B o=\left(\omega_{0} / I_{c}\right)^{2}$ is small compared to unity, where $I_{c}=\sqrt{\gamma / \rho g}$ is the capillary length. Considering typical values $\gamma=10-100 \mathrm{mN} / \mathrm{m}$, the height $h$ of the interface deformation is then on the order of $10-100 \mathrm{~nm}$, illustrating why large beam powers are usually required to detect the induced interface deformation. ${ }^{27}$ The surface tension $\gamma$ can be deduced from the stationary amplitude of the interface deformation (here $h \approx \Pi_{\text {rad }} \omega_{0}^{2} / \gamma$ ) and knowing the tension, the viscosity $\eta$ can be determined from the dynamics. ${ }^{26}$

Except for ultra-low interfacial tension systems where deformed interfaces can be observed with classical microscopy, ${ }^{28}$ interface deformations were usually measured by propagating a probe beam of different optical wavelength and power much smaller than the one of the pump beam. Indeed, the interface deformation induced by the pump beam acts as a partial spherical mirror in reflection (or as a soft lens in transmission) with a focus length proportional to the inverse of the hump curvature $\kappa \cdot{ }^{31}$ Consequently, the induced deformation defocuses the reflected (or focuses the transmitted) probe beam and allows for the measurement of the time-dependent curvature of the hump. ${ }^{25}$ This method was used for the characterisation (i) of the surface tension of water, 25, 32, 33 alcohols, ${ }^{32,} 33$ deposited monolayers, 32,33 and emulsions, $^{34}$ (ii) of the tension of lipid bilayers, ${ }^{35}$ and (iii) of the viscosity of silicone oils. ${ }^{36}$ The technique has the great advantage of rapidity of measurements because the flow triggered by the optical deformation of the interface just extends over the microscopic volume excited by the focused pump beam. Finally, the viscoelastic properties of biological cells 37,38 and natural gels ${ }^{39}$ were also explored; the weak optical absorption of agarose led nonetheless to some thermal issues. This is the main difficulty encountered with this fast contactless optical method: on the one hand focused pump beams are required to overcome the restoring Laplace pressure and reliably detect a lensing effect associated to interface deformation, and on the other hand the minimisation of heating effects demands weak beam focusing.

In order to solve this antagonism, we propose in this investigation to increase the waist sizes and to change the detection method by measuring nanometre-scale deformations, instead of interface curvature. Indeed, probing interface deformations by interferometry enables to have sensitivity with nanometric resolution which offers the great advantage of reducing the pump beam intensity and focusing. Using this new approach, we unambiguously determine surface tension and viscosity, including viscoelasticity when relevant, of non-evaporating and evaporating transparent films of pure liquids, liquid mixtures and suspensions. We also take advantage of evaporation to measure the liquid layer thickness dependence of the deformation time scale in the thin-film regime, ${ }^{40}$ and the viscosity changes related to solute concentration variations. In Section 2, we describe the experimental setup developed for such an investigation. The model for the dynamics of the induced deformations is briefly presented in Section 3 and results for Newtonian films are discussed in Section 4. Section 5 is devoted to the investigation of the rheology of evaporating Newtonian liquid mixtures and suspensions, and viscoelastic behaviours are analysed in Section 6. Finally, Section 7 discusses the influence of weak laser heating, the goal being to enlighten the versatility of optical methods, in terms of actuation and detection, for the investigation of the rheology at small scale when mechanical contact needs to be circumvented.

\section{Experimental setup \& methods}

Our setup, built on a floating optical table, is presented in Fig. 1. A sessile drop (of typical base radius $R \approx 5.0 \mathrm{~mm}$ ) large compared to the used laser beam waist $\left(\omega_{0}=105,175 \mu \mathrm{m}\right)$ is deposited on a prism (see image on the right of Fig. 1a) and locally deformed by the radiation pressure of a green continuous laser (linearly polarised frequency-doubled c.w. $\mathrm{Nd}^{3+}$-YAG laser, Spectra-Physics MILENNIA EV5, wavelength in vacuum $\lambda_{0}=532 \mathrm{~nm}$ ) focused on top of the drop. Experiments are performed at a temperature $T=(20 \pm 1){ }^{\circ} \mathrm{C}$ within an environment of relative humidity $R H \approx 50-60 \%$. Beam powers and waists can be varied using respectively laser control and different lenses; choosing a $f=1 \mathrm{~m}$ focal length for the convergent lens L1 results in $\omega_{0}=175 \mu \mathrm{m}$. Short time exposure is achieved using an electronic shutter (Thorlabs, SHO5 \& $\mathrm{SC} 10)$. The nanoscale deformations induced by the radiation pressure of the pump beam are probed by interferometry using a second loosely-focused low-power continuous red laser (c.w. He-Ne laser, Thorlabs HNL210L-EC, wavelength in vacuum $\lambda_{p}=633 \mathrm{~nm}$ ) from the interferences between the reflection at the top of the drop and some other reflection, chosen as a reference along the optical path (in the present investigation, mainly the glass-air reflection at the exit of the lens $\mathrm{L} 2$, and sometimes the liquid-glass reflection on the prism, see Fig. 1a). The key idea in order to achieve nanometric resolution in interface deformation is to obtain high-contrast 

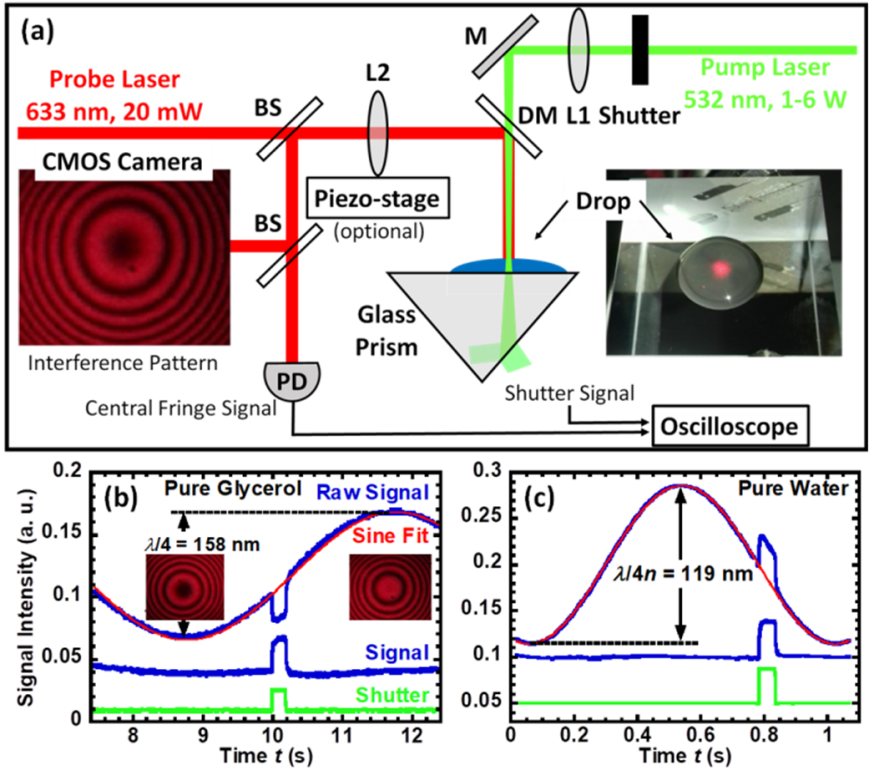

Figure 1: (a) Schematic of the experimental set-up. The radiation pressure of a continuous green pump laser beam is used to locally deform the free surface of a sessile droplet deposited on a prism (right image) and the dynamics of this induced-deformation is probed by interferometry with a loosely focused continuous red He-Ne laser. The photodiode PD measures the dynamics of the intensity at the centre of the fringe pattern produced by the interferences between the reflections of the probe beam at the top of the drop and at the exit face of the lens L2 (left image). The whole fringe pattern can be simultaneously recorded with a CMOS camera to get the dynamics of the drop when it evaporates; another photodiode, not shown, measures the shutter signal actuating the pump exposure. M: mirror, DM: dichroic mirror, BS: beam splitter, L1, L2: lenses. (b) Sine signal intensity of the central fringe induced by the piezoactuation of the lens L2 with the presence of a sudden change when the pump beam is turned on (lower green shutter signal). The temporal evolution of the interface deformation induced by the radiation pressure is deduced from the subtraction of the modulation; the two pictures show respectively the minimum and the maximum intensities at the centre of the fringe pattern. (c) Analogous sine intensity obtained when the optical path difference between the two interfering reflections changes due to the natural evaporation of a droplet of initial volume $30 \mu \mathrm{l}$ (same colour code as in 1b); in the particular case of water, we choose instead the reflexion at the prism-water interface $(n=1.33)$.

Newton's interference fringes as illustrated on the left image of Fig. 1a. At normal incidence (or at quasi-normal incidence, incidence angle $\theta_{i}<5^{\circ}$ ) of the probe beam, the variation from a maximum to a minimum intensity of the central fringe of the interference ring pattern corresponds, in the general case, to a variation of the optical probe path difference of $\lambda_{p} /(4 n)$. To use this variation between the consecutive maximum and minimum intensities as a self-calibrating length, (i) the lens L2 that loosely focuses the probe beam is linearly displaced with a piezo-stage (Thorlabs, NFL5DP20S \& BPC301) along its propagation axis using a saw-teeth signal and, (ii) we measure the dynamics of the central fringe of the pattern formed by the interference between the reflection at the top of the drop and that at the moving exit surface of the piezo-actuated lens L2; note that the lens L2 does not have any anti-reflection coating to increase the fringe contrast (see Fig. 1a). Then, the intensity $I(t)$ measured at the centre of the fringe pattern displays a temporal sine modulation of amplitude $\lambda_{p} / 4$ ( $n=1$ in air) and temporal variation dictated by the chosen displacement velocity of the lens L2. Any actuation of the drop interface by the radiation pressure changes the optical path, modifies the intensity of the central fringe which, as such, contains the temporal signature of the induced deformation. This general method is particularly suitable for non-evaporating (such as glycerol) or turbid liquids or when substrates are opaque or rough. An example of temporal variation of the central intensity of the fringe pattern in the absence and presence of radiation pressure is illustrated in Fig. $1 \mathrm{~b}$ for a pure glycerol drop. In the absence of pump beam, a sine variation is observed resulting from the piezo-actuated displacement of the lens L2. When the pump beam is turned on with the shutter (lower green signal in Fig. 1b), the optical path difference between the probe reflection at lens $L 2$ and the one on top of the drop suddenly changes and modifies the central intensity. The subtraction of the sine modulation eventually gives the temporal evolution of the interface deformation driven by the radiation pressure of the pump beam (lower blue curve in Fig. 1b called 'signal'); an enlarged view is presented in Fig. 2 dedicated to the quantitative investigation of the rheology of glycerol. Note that a laser exposure of a few hundreds of milliseconds is sufficient to capture the whole dynamics (including the steady state), due to the smallness of the excited area, offering in situ and fast characterization.

When liquids evaporate (Fig. 1c), the piezo-actuation of the lens L2 is no more required because natural evaporation decreases the droplet thickness and then continuously increases the optical path between the probe beam reflection at lens L2 and the one at the drop interface. Then the intensity $I(t)$ at the centre of the central fringe naturally oscillates and these oscillations serve for determining the direction and magnitude of the induced interface deformation; ${ }^{41}$ this case is described in details in Fig. 3 dedicated to the rheological investigation of water thin-films. Note that in the particular case of water, we can choose the reflexion at the prism-water interface, instead at $L 2$, as a reference since water is highly transparent at the probe beam wavelength and its index of refraction remains constant during evaporation; this corresponds to what we did in Fig. 1b. Finally, we are also able to capture the entire Newton ring pattern with a CMOS camera (Thorlabs DCC1645C, $25 \mathrm{fps}$ ) in order to measure the dynamics of the local topography of the free surface of the drop over long time. According to the classical Newton ring method, ${ }^{42}$ the dynamics of the radii of the successive rings (illustrated in Fig. 1a, left image, and Fig. 1b) indeed provides access to the time variation of the curvature of a sessile drop during its evaporation and then to its dynamical height and evaporation rate if the contact line remains fixed; ${ }^{41}$ note that replacing the photodiode by a fast camera with the same temporal resolution would allow getting the dynamics of the entire interface deformation, beyond its maximum amplitude measured at the centre of the fringe pattern.

\section{Dynamics of interface deformations in the viscous regime}

The dynamics of small-amplitude deformations of free surfaces by the radiation pressure has been already described theoretically for semi-infinite transparent liquid layers 24,43 and experimentally tested with acoustic waves. ${ }^{24}$ In brief, it is deduced from the linearised unsteady Stokes equations, mass conservation and stress balance at the free-surface. For smallamplitude deformations, characterized by $h / \omega_{0}<<1$, the 
curvature can be approximated by a Laplacian form and the radiation pressure can be estimated at normal incidence. The stress balance at the interface is then described by:

$$
\rho g h(r, t)-\gamma \frac{1}{r} \frac{\partial}{\partial r}[r h(r, t)]-2 \eta \frac{\partial v_{z}}{\partial z}(r, t)=\Pi(r, t),
$$

where $v_{z}(r, t)$ is the vertical velocity of the liquid and:

$$
\Pi(r, t)=\Pi_{0} \exp \left(-2 \frac{r^{2}}{\omega_{0}^{2}}\right) H(t)
$$

is the space-dependent radiation pressure acting on the interface at normal incidence from time $t=0 \quad(H$ is the Heaviside distribution). The amplitude of the radiation pressure on the beam axis when the pump beam is incident from the air is given by:

$\Pi_{0}=\frac{2}{c}\left(\frac{n-1}{n+1}\right) \iota_{0}$.

Finally, for small-amplitude deformations, the height variation is obtained from mass conservation, $h(r, t) \simeq \int_{0}^{t} v_{z}\left(r, z=0, t^{\prime}\right) d t^{\prime}$.

The present investigation is devoted to the rheology of viscous fluids, expected when gravito-capillary waves are damped by the viscous dissipation, i.e. when:

$$
\sqrt{\frac{\gamma}{\rho} k^{3}\left(1+\frac{\rho g}{\gamma k^{2}}\right)}<<\left(\frac{\eta}{\rho} k^{2}\right)
$$

Since the beam waist $\omega_{0}$ is the characteristic radial extension of the Gaussian distribution of the radiation pressure, we define a characteristic wavenumber as $k_{c}=1 / \omega_{0}$; the corresponding optical Bond number is $B o=\left(k_{c} I_{c}\right)^{-2}=\left(\omega_{0} / I_{c}\right)^{2}$. With these notations, and for a time $t \gg\left(\eta k^{2} / \rho\right)^{-1}$, we finally find the expected viscous dynamics of the interface deformation and of its relaxation after an exposure time $t_{0}:{ }^{24}$

$$
\left\{\begin{array}{r}
h\left(r=0, t \leq t_{0}\right)=\frac{\Pi_{0}}{4 \gamma k_{c}^{2}} \int_{0}^{\infty} \frac{\exp \left(-\tilde{k}^{2} / 8\right)}{B o+\tilde{k}^{2}}[1-\exp (-\Omega(\tilde{k}) t)] \tilde{k} d \tilde{k} \\
h\left(r=0, t \geq t_{0}\right)=\frac{\Pi_{0}}{4 \gamma k_{c}^{2}} \int_{0}^{\infty} \frac{\exp \left(-\tilde{k}^{2} / 8\right)}{B o+\tilde{k}^{2}}\left[1-\exp \left(-\Omega(\tilde{k}) t_{0}\right)\right] \\
\quad \times \exp (-\Omega(\tilde{k}) t) \tilde{k} d \tilde{k}
\end{array}\right.
$$

where $\tilde{k}=k / k_{c}$ is the dimensionless wavenumber and $\Omega(\tilde{k})$ is the rate of evolution of the overdamped mode $\tilde{k}$. In the thicklayer case considered above $\left(h_{0} k_{c}=h_{0} / \omega_{0} \gg>1\right)$, one has:

$\Omega(\tilde{k})_{\text {Thick }}=\left[\frac{\gamma}{\rho} k^{3}\left(1+\frac{\rho g}{\gamma k^{2}}\right)\right] /\left[2\left(\frac{\eta}{\rho} k^{2}\right)\right]=\frac{1}{\tau_{\text {Thick }}} \tilde{k} \frac{\left(1+B o / \tilde{k}^{2}\right)}{1+B o}$

which allows to define a characteristic damping time associated to the mode $k_{c}$ :

$\tau_{\text {Thick }}=\frac{2 \eta}{\gamma k_{c}}(1+B o)^{-1}=\frac{2 \eta \omega_{0}}{\gamma}(1+B o)^{-1}$.

In the opposite thin-layer case $\left(h_{0} k_{c}=h_{0} / \omega_{0}<<1\right)$, and more generally whatever the height $h_{0}$ is, a full theory of radiation pressure effects in presence of finite-distance boundaries is still missing. Nonetheless, at scales below the capillary length $I_{c}$, the overdamped dynamics of purely viscous
Newtonian films in the linear regime of small deformation is now well-established and shows that: ${ }^{44}$

$\Omega(k)=\frac{\gamma}{4 \eta} k\left[\frac{\sinh \left(2 k h_{0}\right)-2 k h_{0}}{\cosh ^{2}\left(k h_{0}\right)+\left(k h_{0}\right)^{2}}\right]$,

in the absence of slip at the substrate. Then, the thickness $h_{0}$ emerges as an axial length scale for the 'thin' liquid layer case ( $\left.h_{0} k_{c}<<1\right)$, leading to:

$\Omega(k)_{\text {Thin }} \sim \frac{\gamma h_{0}^{3}}{3 \eta} k^{4}$.

Consequently, assuming the same weak corrections in Bo number, we write:

$\Omega(\tilde{k})_{\text {Thin }}=\frac{\gamma h_{0}{ }^{3} k_{c}^{4}}{3 \eta} \tilde{k}^{4}\left(1+\frac{B o}{\tilde{k}^{2}}\right)=\frac{1}{\tau_{\text {Thin }}} \tilde{k}^{4} \frac{\left(1+B o / \tilde{k}^{2}\right)}{1+B o}$,

and deduce a new characteristic time scale:

$\tau_{\text {Thin }}=\frac{3 \eta}{\gamma h_{0}{ }^{3} k_{c}{ }^{4}}(1+B o)^{-1}=\frac{3 \eta \omega_{0}{ }^{4}}{\gamma h_{0}{ }^{3}}(1+B o)^{-1}$,

which shows the cross-dependence in both $\omega_{0}$ and $h_{0}$.

Finally, as illustrated by Eq. 5 , the same time scales $\tau_{\text {Thick }}$ and $\tau_{\text {Thin }}$ are expected to describe both the interface deformation ant its relaxation after shutting down the laser. These predicted dynamics are confronted to various experimental situations in the following sections.

\section{Optorheology of transparent liquid films}

As a first example we investigate the rheology of a nonevaporating 'thick' viscous film. We consider a sessile drop of pure glycerol (ACROS 99.8\%), $1 \mathrm{~mL}$ drop volume and $1 \mathrm{~mm}$ height, and use the piezo-actuated lens scheme to modulate temporally the intensity $I(t)$ measured at the centre of the fringe pattern. As glycerol is highly hygroscopic, the experiment is performed just after drop deposition and stabilisation. The extraction of the temporal evolution of the nanometric interface deformation induced by the radiation pressure of the pump beam (Fig. 1b) is presented in Fig. 2 for an exposure time of $200 \mathrm{~ms}, P=3 \mathrm{~W}$ and the two beam waists values $\omega_{0}=105,175 \mu \mathrm{m}$. As expected from Eq. 5 for a given power $P$, the highest deformation is obtained for the smallest beam-waist corresponding as well to the smallest Bond number Bo. This can easily be retrieved analytically when looking at steady-state deformations for which $h(0, t \rightarrow \infty)_{B o<<1} \sim P . \ln [8 /(\delta . B o)]$, where $\delta=1.781$ is the Euler constant. ${ }^{28}$ Moreover, according to Eqs. 5-7, the fit of the growing deformation gives the amplitude $\Pi_{0} \omega_{0}^{2} / 4 \gamma$, the time scale $\tau_{\text {Thick }}$ and the Bo number value. From the amplitude we get $\gamma$ when $n$ is known, from the measured time scale $\tau_{\text {Exp }}$ (here corresponding to $\tau_{\text {Thick }}$ ) we determine the viscous velocity $\gamma / \eta$ and then $\eta$, and from Bo we can deduce $\rho / \gamma$, and thus $\rho$. Using $n=1.474$ and $\rho=1.261 \mathrm{~g} / \mathrm{cm}^{3}$ for glycerol at $20^{\circ} \mathrm{C}$ and taking into account the height resolution of $\pm 1 \mathrm{~nm}$ on the one hand and the relative error $d \tau_{\text {Exp }} / \tau_{\text {Exp }}=1 \%$ deduced 


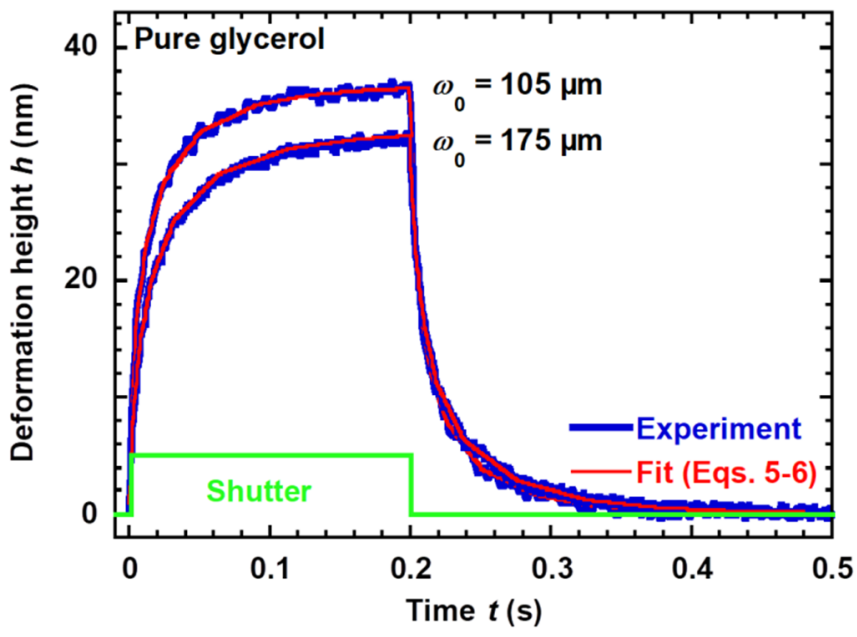

Figure 2: Time-resolved interface deformation induced by the radiation pressure on a glycerol drop in the thick-film regime, for $P=3 \mathrm{~W}$ and two values of the beam waist $\omega_{0}$. The green signal represents the laser exposure window. As glycerol does not evaporate, the piezo-actuation of the lens $L 2$ is used to get the reference fringe pattern signal (Fig. 1b). The continuous red lines are data fits according to Eqs. 5-6 that gives the values of the amplitude $\Pi_{0} \omega_{0}^{2} / 4 \gamma$, the time scale $\tau_{\text {Thick }}$ and the optical Bond number Bo as a tiny correction.

from the standard deviation on the other hand, we finally find $\gamma=(65 \pm 2) \mathrm{mN} / \mathrm{m}$ and $\eta=(1.1 \pm 0.05)$ Pa.s, in very good agreement with literature $(\gamma=63.4 \mathrm{mN} / \mathrm{m}$ and $\eta=1.07$ Pa.s at $\left.20^{\circ} \mathrm{C}\right) .45$ The dynamics of deformation predicted for the 'thick' layer case $\left(h_{0} / \omega_{0}>>1\right)$ is thus quantitatively retrieved experimentally.

As a second example, we investigated the rheology of an evaporating deionised (DI) water drop. Inasmuch water is transparent and its properties do not evolve with evaporation, we choose as a reference the reflexion of the probe beam at the dropprism interface, instead of the reflexion at $L 2$, in order to show the versatility of the method. Since a single measurement spans a few hundreds of milliseconds, a unique advantage of our optorheology approach is the possibility to perform a series of measurements in a row in non-equilibrium conditions, such as evaporation which takes place over a much larger time scale than the characteristic time of interface deformation $\tau_{\text {Thick,Thin }}$. Note also that the rheology of water, normally considered as an inertial liquid, can be analysed using a low-Reynolds-number point of view because viscous dissipation increases drastically in the thin-layer case due to $\left(h_{0} k\right)^{3} \sim\left(h_{0} / \omega_{0}\right)^{3} \rightarrow 0$. Then it becomes possible to investigate the 'thin' layer rheology $\left(h_{0} / \omega_{0}<<1\right)$ of a water droplet in order to observe the expected increase of $\tau_{\text {Thin }}$ related to the $1 / h_{0}{ }^{3}$ dependence (see Eq. 11). To do so, we first need to keep constant the ambient humidity, here $R H \approx 50 \%$, and then to calibrate the rate of natural evaporation of a first drop in these environmental conditions. Then, we use the Newton ring pattern produced by the probe beam reflections to measure the curvature $\mathbb{C}(r=0, t)$ on top of the drop deposited on the prism. In presence of evaporation, this Newton ring pattern evolves with time and the measurement of its evolution gives access to the dynamics of the drop height $h_{0}(t)$ if the contact line of the deposited drop, of radius $R$, remains fixed at all times; this last condition is always verified in our experiments. The shape of the drop deposited on the prism depends nonetheless on the relative importance of gravity on surface tension effects measured by the hydrodynamic Bond number $B o_{H}=\left(R / I_{c}\right)^{2}$. When $B o_{H}<<1$, the drop shape coincides exactly with a spherical cap and $\mathbb{C}(0, t)=4 h_{0}(t) /\left(R^{2}+h_{0}(t)^{2}\right)$. Unfortunately, the optical coating of the prism prevented us from confidently depositing regular sessile drops with $B o_{H}<<1$. We thus choose an initial volume $V_{0}=30 \mu \mathrm{l}$ and $R=4.5 \mu \mathrm{m}$ for the investigation of the rheology of aqueous solutions. For DI water $B o_{H} \approx 2.76\left(I_{c} \approx 2.71 \mathrm{~mm}\right)$ and the shape of the drop is slightly distorted by gravity. With our drop size, the contact angle $\theta$ on glass remains weak so that the drop height $h(r, t)$, the curvature $\mathbb{C}(r, t)$ and its volume $V(t)$ can be described by the following expressions: 46

$$
\left\{\begin{array}{c}
h(r, t) \approx I_{c}\left[\frac{I_{0}\left(R / I_{c}\right)-I_{0}\left(r / I_{c}\right)}{I_{1}\left(R / I_{c}\right)}\right] \theta(t), \\
\mathbb{C}(r, t) \approx \frac{1}{I_{c}}\left[\frac{I_{0}\left(r / I_{c}\right)}{I_{1}\left(R / I_{c}\right)}\right] \theta(t), \\
V(t) \approx \pi I_{c}^{3}\left[\frac{I_{0}\left(R / I_{c}\right)}{I_{1}\left(R / I_{c}\right)}\left(\frac{R}{I_{c}}\right)^{2}-2\left(\frac{R}{I_{c}}\right)\right] \theta(t),
\end{array}\right.
$$

where $I_{0}(x)$ and $I_{1}(x)$ are zeroth- and first-order modified Bessel functions. Then, by measuring the curvature $\mathbb{C}(0, t)$ on top of the drop with the Newton ring pattern evolution, we easily deduce the drop height variation due to evaporation from the ratio $h_{0}(t) / \mathbb{C}(0, t)$ and the corresponding variation of the drop volume $V(t) / h_{0}(t)$. Note that $B o_{H}$ numbers of the order of a few unities allow expanding these ratios in Taylor series:

$$
\left\{\begin{array}{l}
\frac{h_{0}(t)}{\mathbb{C}(0, t)} \approx \frac{R^{2}}{4}\left[1+\frac{B o}{16}+\frac{B o^{2}}{576}+O\left(B o^{4}\right)\right] \\
\frac{V(t)}{h_{0}(t)} \approx \frac{\pi R^{2}}{2}\left[1+\frac{B o}{48}+\frac{B o^{2}}{2304}+O\left(B o^{3}\right)\right]
\end{array}\right.
$$

which show that the drop shape can be described in terms of corrections to the spherical cap case at small wetting angle (i.e. $\quad h_{0}(t) / \mathbb{C}(0, t) \approx R^{2} / 4$ and $V(t) / h_{0}(t) \approx \pi R^{2} / 2$ when $\left.\left(h_{0}(t) / R\right)^{2}<<1\right)$. For a water drop of initial volume $V_{0}=30 \mu \mathrm{l}$ and radius $R=4.5 \mu \mathrm{m}$, we find $h_{0}=895 \mu \mathrm{m}$ and the Inset of Fig. 3a, shows that $h_{0}(t)$ decreases linearly with time during natural evaporation according to $d h_{0} / d t=-(149 \pm 1) \mathrm{nm} / \mathrm{s}$; the measured uncertainty on $h_{0}$, extracted from both Newton 

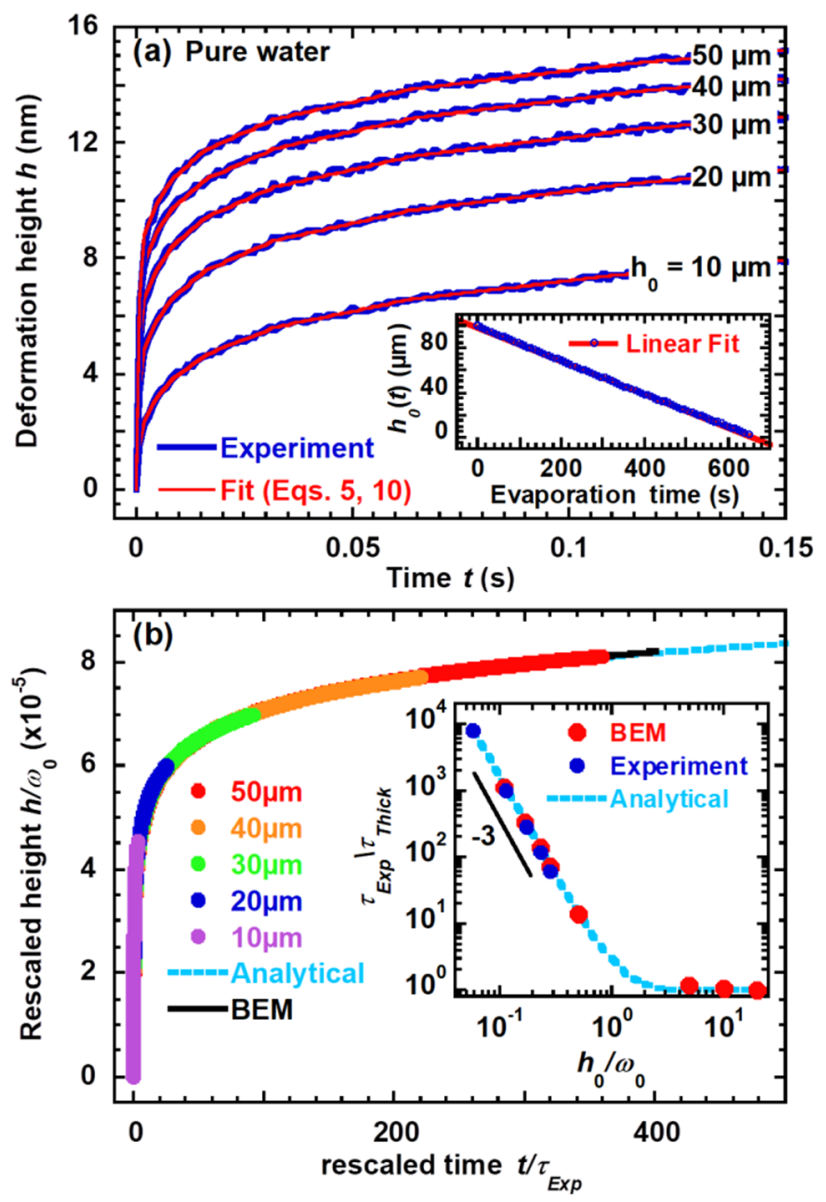

Figure 3: (a) Time-resolved interface deformation of an evaporating DI water drop in the thin-film regime obtained for heights $h_{0}$ decreasing from 50 to $10 \mu \mathrm{m}$ ( $\pm 1 \mu \mathrm{m}$ uncertainty on $h_{0}$ from the measured evaporation rate, shown in the Inset for the range of investigated heights); $P=3 \mathrm{~W}, \omega_{0}=175 \mu \mathrm{m}$ and $150 \mathrm{~ms}$ exposure time; the continuous red lines are data fits according to Eqs. 5 , 10. (b) Collapse of the dynamic deformation data obtained for different heights $h_{0}$ onto a thin-film master behaviour when time is rescaled with $\tau_{\text {exp }}$. Inset: experimental illustration of the $h_{0}^{-3}$ dependence of the measured time scale $\tau_{\text {Exp }}$, supposed to represent $\tau_{\text {Thin }}$, and confirmation by an analytic calculation and numerical BEM simulations; the analytic solution is obtained by solving Eqs 5, 8 to show the 'thin-thick' layer crossover in $h_{0} / \omega_{0}$.

ring tracking analysis and the measured base radius $R$ of the deposited drop, is $\pm 1 \mu \mathrm{m}$. Fig. 3 illustrates the use of this strategy on a single DI water droplet with optical actuation performed at evaporation times corresponding to the expected drop heights for $P=3 \mathrm{~W}$ and $\omega_{0}=175 \mu \mathrm{m}$. While the stationary amplitude of the deformation should not be affected (it solely depends on $P, \omega_{0}, n, \rho$ and $\gamma$ that do not vary during the experiment), one can observe in Fig. 3a a significant slowing down of the dynamics of deformation when decreasing $h_{0}$. Measurements of the time scale $\tau_{\text {Exp }}$ as a function of $h_{0}$ are presented in the Inset of Fig.3b; the error from the standard deviation is again $d \tau_{\text {Exp }} / \tau_{\text {Exp }}=1 \%$. They clearly provide evidence for the $h_{0}^{-3}$ behaviour expected from the expression of $\tau_{\text {Thin }}$. This behaviour is quantitatively confirmed analytically by solving Eqs. 5, 8 and numerically by using the Boundary Element Method 47 with reference data ( $\gamma=72 \mathrm{mN} / \mathrm{m}$ and $\eta=1.0310^{-3}$ Pa.s at $20^{\circ} \mathrm{C}$ ). ${ }^{45}$ Moreover, Fig.
$3 \mathrm{~b}$ illustrates the expected 'thin' layer scaling by the quantitative collapse of the whole set of dynamic deformations, obtained for different heights $h_{0}$, onto a single master behaviour predicted analytically by Eqs. 5, 10, 11 and retrieved numerically when time is rescaled by $\tau_{\text {Exp }}=\tau_{\text {Thin }}$.

\section{Optorheology of a transparent Newtonian liquid mixture \& suspension films}

Since radiation pressure effects offer the opportunity to catch 'instantaneous' properties of out-of-equilibrium processes characterized by much longer time scales, we first extend the use of evaporation to investigate the dependence in solute concentration of the viscosity of evaporating liquid mixtures.

As a first example, we choose a DI water-glycerol mixture of initial glycerol concentration $C_{0}=3 \% \mathrm{wt}$; the initial volume is $V_{0}=30 \mu \mathrm{l}$, the drop radius at contact on the prism is $R=4.5 \mathrm{~mm}$ and the chosen exposure time to radiation pressure is $150 \mathrm{~ms}$. As water evaporates, the glycerol mass fraction $C$ continuously increases leading to variations in density, viscosity, index of refraction and surface tension of the mixture. We choose low humidity environment conditions to keep a linear decrease of $h_{0}$ with time and reach large glycerol concentrations; beyond $C \simeq 90 \% \mathrm{wt}$, the evaporation of water is expected to asymptotically stop as glycerol is highly hygroscopic. Due to the continuous variation of the index of refraction $n$ of the mixture related to its evaporation, we performed measurements using interferences between the reflection at the top of the drop and the one at the exit surface of the lens L2. As the optical stress of the pump beam depends on the index contrast (see Eq. 3), we need nonetheless to properly estimate $n(t)$. To do so, we first measure the evaporation rate of a water-glycerol drop using the Newton ring method. However, due to water evaporation, the capillary length evolves with time from that of water $\left(I_{c} \approx 2.71 \mathrm{~mm}\right)$ to that of glycerol $\left(I_{c} \approx 2.26 \mathrm{~mm}\right)$, and the Bond number $B o_{H}$ increases from 2.76 to 3.96 . So we choose deducing the height variation $h_{0}(t)$ from the curvature using a mean value of $B o_{H}=3.36 \pm 0.60$ in Eq. 13 and considering the maximum deviation from this mean value as an uncertainty in the determination of $h_{0}(t)$ and $V(t) ; h_{0}(t)$ and $V(t)$ are then determined with respective additional uncertainties of $5 \%$ and $6 \%$. We thus find $d h_{0} / d t=-(105 \pm 7) \mathrm{nm} / \mathrm{s}$ from which we deduce the time variation of the volume $V(t)$ of the evaporating drop as a function of $h_{0}(t)$. Then, we use the property that water-glycerol mixtures behave as regular solutions, with a precision better that $2 \% .{ }^{48}$ The concentration can thus be written as $c\left(h_{0}(t)\right)=\rho_{G} V_{G} /\left[\rho_{G} V_{G}+\rho_{w}\left(V(t)-V_{G}\right)\right]$, where $\rho_{i=G, W}$ and $V_{G}$ are respectively the density and volume of glycerol (G) and water (W). Knowing the expression of $C\left(h_{0}\right)$ , we use an empiric variation of the index of refraction $n(C)=1.333(1-C)+1.474 C$ (known to present a deviation 

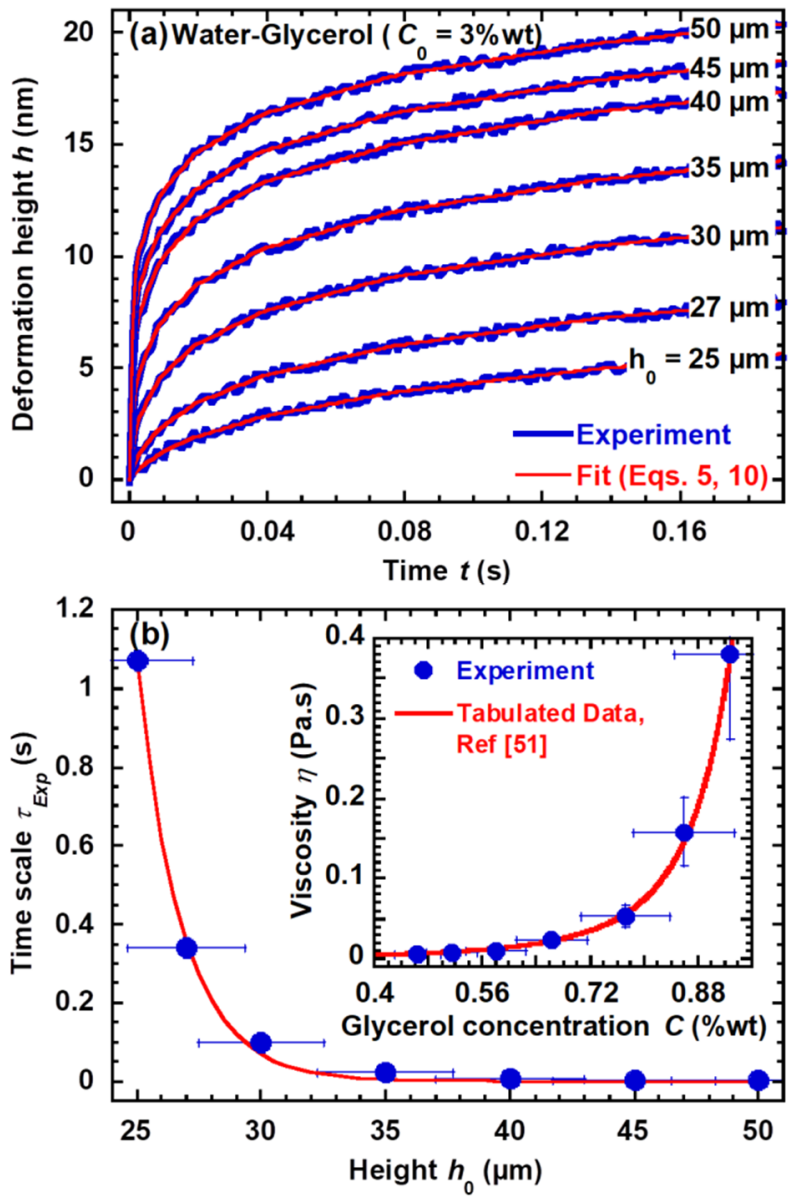

Figure 4: (a) Time-resolved interface deformation of an evaporating DI waterglycerol drop of initial concentration $C_{0}=3 \%$ wt in the 'thin' film regime for different heights $h_{0}$ varying from 50 to $25 \mu \mathrm{m}$ (uncertainty $\pm 1 \mathrm{~nm}$ on $h$ from interferometric measurement and $\pm 6 \mu \mathrm{m}$ on $h_{0}$ deduced from the evaporation rate). The used power and waist are $P=3 \mathrm{~W}$ and $\omega_{0}=175 \mu \mathrm{m}$. The continuous red lines are data fits according to Eqs. 5, 10. (b) Corresponding time scale $\tau_{\text {Thi }}$ variation with the thickness $h_{0}$; the continuous line is a guide for the eye. Inset: resulting variation of the viscosity $\eta(C)$ with the increase in glycerol concentration $C$ due to water evaporation; the continuous line shows tabulated data from the literature. ${ }^{51}$

smaller that $0.4 \%)^{49}$ to quantify the optical stress. Also, as the optical Bond number is larger than $410^{-3}$ (pure water) and smaller than $610^{-3}$ (pure glycerol), it behaves as a very weak correction to unity in Eqs. 7 and 11, and we consider a mean constant value $510^{-3}$. Finally, as the initial sample is mostly composed of water, we choose large enough evaporation times to work with 'thin' sessile drops and preserve a viscous dynamics for all measurements. Using these conditions, we are able to deduce the surface tension $\gamma(C)$ from the amplitude of the deformation and the viscosity $\eta(C)$ from its dynamics. Fig. 4a illustrates the dynamics of deformation measured for different heights $h_{0}$. Once again, the dynamics of deformation displays a slowing down when decreasing the drop height. This decrease is expected to be due to both the $h_{0}^{-3}$ dependence in the time scale of the 'thin' film regime and the viscosity increase with glycerol concentration $\eta(C)$ in $\tau_{\text {Thin }}$ (Eq. 11); note that contrary to pure DI water experiments, the amplitude of deformation (Eq. 5) now varies with $h_{0}$ since
$\gamma=\gamma(C)$ and $n=n(C)$ with $C\left(h_{0}\right)$ due to water evaporation. Fig. $4 \mathrm{~b}$ presents the variation of the measured time scale $\tau_{\text {Exp }}$ with the height $h_{0}$ of the drop. We deduce weakly fluctuating values of $\gamma(C)$ around a mean value $6710^{-3} \mathrm{Nm}^{-1}$ without any decreasing trend of $\gamma(C)$ from $69.510^{-3} \mathrm{Nm}^{-1}$ (glycerol $46 \% \mathrm{wt}$ ) to $66.510^{-3} \mathrm{Nm}^{-1}$ (glycerol $92 \% \mathrm{wt}$ ). ${ }^{49}$ This absence of trend is not necessarily surprising since the $310^{-3} \mathrm{Nm}^{-1}$ difference leads to a relative variation $d \gamma / \gamma=5 \%$ which is in agreement with the $\pm 1 \mathrm{~nm}$ resolution in height of our setup; on the one hand Eq. 5 predicts $d h / h \approx d \gamma / \gamma$ and on the other hand $d h / h \geq 1 / 20=5 \%$ (see Fig. 4a). We thus confidently used $\gamma(c)=6710^{-3} \mathrm{Nm}^{-1}$ to further deduce the viscosity dependence $\eta\left(h_{0}\right)$ from Fig. $4 \mathrm{~b}$. This mean value of the surface tension also suggests that water evaporation does not produce significant glycerol concentration gradient at the drop interface. Considering the mutual diffusion coefficient $D_{W-G}=2.1410^{-10} \mathrm{~m}^{2} / \mathrm{s} 50$ at $25^{\circ} \mathrm{C}$ for a glycerol concentration of $70 \% \mathrm{wt}$ (in the middle of our range of investigation, see Fig. $4 \mathrm{~b})$, the diffusion 'velocity' over the drop height $h_{0} \approx 35 \mu \mathrm{m}$ associated to this concentration (see Fig. 4a) is $D_{w-G} / h_{0} \approx 6 \mu \mathrm{m} / \mathrm{s}$, which is much larger than the measured evaporation rate $\left|d h_{0} / d t\right| \approx 0.1 \mu \mathrm{m} / \mathrm{s}$. Consequently, diffusion dominates evaporation and ensures homogenisation of the concentration within the evaporating mixture. So, using the expected variation $C\left(h_{0}\right)$ and $\tau_{\text {Exp }}=\tau_{\text {Thin }}$, we finally end up with the variation $\eta(C)$ illustrated in the Inset of Fig. $4 \mathrm{~b}$. The error on $C\left(h_{0}\right)$ is given by: $d C=|\partial C / \partial V| d V=\left(\frac{\rho_{W}}{\rho_{G} V_{G}}\right) C^{2} \frac{\partial V}{\partial h_{0}}\left(6 \% h_{0}+d h_{0}\right)$, with $V_{G}=3 \% \rho_{w} V_{0} /\left(97 \% \rho_{G}+3 \% \rho_{w}\right), V_{0}=30 \mu \mathrm{L}$ and $\partial V / \partial h_{0}=V / h_{0}$ (Eq. 13); the error on the viscosity measurement, deduced from Eq. 11 , is given by $d \eta / \eta=d \tau_{\text {Thin }} / \tau_{\text {Thin }}+3 d h_{0} / h_{0}$. The variation of $\eta(C)$ is in quantitative agreement with literature data illustrated by the continuous line. ${ }^{51}$

Our second example is motivated by probing the efficiency of optorheology in turbid solutions. To do so, we investigate the rheology of a suspension of polystyrene latex beads (negatively-charged Estapor ${ }^{\circledR}$ microspheres in an aqueous solution, diameter $\varnothing=100 \mathrm{~nm}$, initial concentration $10 \%$ wt of solid content) diluted in DI water to reach a concentration of $\Phi_{0}=0.25 \% \mathrm{v} / \mathrm{v}$. The initial volume is $V_{0}=30 \mu \mathrm{l}$ and the drop radius on the prism is $R=4.5 \mathrm{~mm}$. The measurement procedure is exactly the same as that used for the waterglycerol mixture. The turbidity of the solution (see picture in Fig. 5) indeed imposes the analysis of interferences formed between the probe reflection at the interface of the evaporating drop suspension and another reference in air, here the reflection on lens L2. Moreover, as turbidity is a bulk property, it does not influence radiation pressure effects that are sensitive to the refractive index contrast at the drop interface. Finally, since water is the major initial constituent, we will assume a water-air interface and continue to investigate 'thin' drops to analyse interface deformation in the viscous regime. Fig. 5 illustrates several dynamics obtained for different thicknesses $h_{0}$ expected from the evaporation rate 


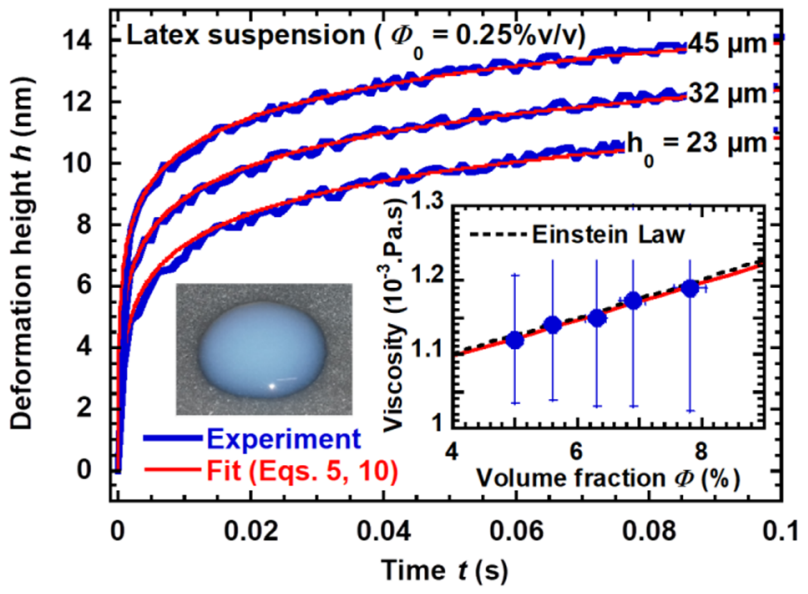

Figure 5: Time-resolved interface deformation of an evaporating aqueous suspension of latex nanospheres (diameter $\varnothing=100 \mathrm{~nm}$, initial concentration $\Phi_{0}=0.25 \% \mathrm{v} / \mathrm{v}$, note the turbidity of the drop solution) in the 'thin' film regime for three heights $h_{0}( \pm 1 \mu \mathrm{m})$; the continuous red lines are data fits according to Eqs. 5,10 . Inset: corresponding variation of the viscosity $\eta(\Phi)$ with the increase in nanosphere concentration due to water evaporation; the Einstein prediction at low concentration is also illustrated for comparison.

of DI water. The Inset of Fig. 5 finally presents the deduced viscosity variations with the volume fraction of latex beads $\eta(\Phi)$ when we assume that the surface tension and the index of refraction of the suspension remain constant during evaporation and equal to those of water due to the low concentration in nanospheres; errors are $d \eta / \eta=d \tau_{\text {Thin }} / \tau_{\text {Thin }}+3 d h_{0} / h_{0}, \quad d \Phi=|\partial \Phi / \partial V| d V=\frac{\Phi^{2}}{V_{0} \Phi_{0}} \frac{\partial V}{\partial h_{0}} d h_{0} . \quad$ A linear fit leads to $\eta(\Phi)=0.998 \cdot(1+2.486 \cdot \Phi) 10^{-3}$ Pa.s in very close agreement with the Einstein law, illustrated as well in the Inset of Fig. 5. While appealing, the investigation of viscosity variations at large concentration is made difficult by the increasing number of beads adsorbed at the drop interface that modifies radiation pressure effects.

\section{Optorheology of transparent polymer gel films}

Since most soft matter systems are not Newtonian, it is also of importance to check whether or not radiation pressure can be used to probe elastic and viscoelastic properties. We choose to work with a solution composed of small molecular weight PolyAcrylAmide (PAAm, 5-6 M, Polysciences) simply diluted at $C_{0}=2 \% \mathrm{wt}$ in DI water. The initial volume is $V_{0}=30 \mu \mathrm{l}$ and the drop radius at contact on the prism is $R=4.5 \mathrm{~mm}$. Considering the expected value of the viscosity, the experiment is performed in the 'thick' layer regime ( $h_{0} \simeq 895 \mu \mathrm{m}$ ) just after drop deposition and stabilisation. As PAAm solutions neither absorb nor scatter light when well mixed, we use the same procedure as for water film measurements: we analyze interferences between the probe reflection from the mixtureprism surface, used as reference, and the reflection at the mixture-air interface in presence of water evaporation. Fig. 6 illustrates the dynamics of deformation during an exposure time to radiation pressure of $50 \mathrm{~ms}$ followed by the relaxation of the interface after the laser is turned off. This signal clearly shows a very different behaviour compared to previous ones obtained for Newtonian liquids. Upon optical loading by the radiation pressure, we observe a step-like increase of the height, signature of the material elasticity, followed by a slow continuous increase which indicates a viscoelastic behaviour with a flow of the material at longer times. The same behaviour is observed in reverse after turning off the laser; note that no permanent interface deformation is observable.

Models describing interface deformation by localized radiation pressure in viscoelastic systems are scarce. ${ }^{37,}{ }^{39}$ We thus propose modelling optorheology using a one-dimensional approach. We assimilate the load $\sigma_{0}$ as radially constant and equal to half of the optical stress at the pump beam centre ( $\sigma_{0}=\Pi_{0} / 2$ ), since the drop is deposited on the glass prism and then stretched only in one direction (see schematics in Fig. 6). Moreover, considering the observed dynamics, we choose to confront our measurements to the well-known Standard Linear Solid model in its 'Voigt' form. ${ }^{52}$ It is known to fairly reproduce the weakly nonlinear rheological response of 'real' materials when no permanent strain is left. This model couples pure elasticity (here of Young modulus $E_{1}$ ), mimicking the observed instantaneous stretching upon optical loading, to a Kelvin-Voigt unit (with elasticity $E_{2}$ and viscosity $\eta$ ) that could describe the following slow viscoelastic deformation. Writing the strain $\varepsilon(t)=h(t) / h_{0}$ and considering the viscoelastic time scale $\tau_{v}=\eta / E_{2}$ in this $1 \mathrm{D}$ description, the expected dynamics of the strain during optical loading $\varepsilon_{\uparrow}(t)$ and relaxation $\varepsilon_{\downarrow}(t)$ after an exposure time $t_{0}$ are given by: ${ }^{52}$

$$
\left\{\begin{array}{l}
\varepsilon_{\uparrow}\left(t \leq t_{0}\right)=\frac{\sigma_{0}}{E_{1}}+\frac{\sigma_{0}}{E_{2}}\left(1-\exp \left(-t / \tau_{v}\right)\right), \\
\varepsilon_{\downarrow}\left(t \geq t_{0}\right)=\frac{\sigma_{0}}{E_{2}} \exp \left(-t / \tau_{v}\right)\left[\exp \left(t_{0} / \tau_{v}\right)-1\right] .
\end{array}\right.
$$

As Illustrated in Fig. 6, data are reasonably well fitted by this simplified description with some discrepancies in the relaxation. We use $P=3 \mathrm{~W}$ and $\omega_{0}=175 \mu \mathrm{m}$ for the pump beam, and a refractive index $n=1.38{ }^{53}$ for the chosen PAAm concentration, leading to an optical load value $\sigma_{0}=0.033 \mathrm{~Pa}$. We get the elastic modulus $E_{1}=h_{0} \sigma_{0} / h$ from the first fast increase of the rising deformation $\left(E_{1}=2214 \mathrm{~Pa}\right)$ and from its fast relaxing part $\left(E_{1}=2200 \mathrm{~Pa}\right)$. Both values are very close, leading to a mean value $E_{1}=(2205 \pm 175) \mathrm{Pa}$; $d E_{1} / E_{1}=d h / h \simeq 8 \%$ in the elastic part of the signal. The fit of the amplitude of the slow variation of the rising signal according to Eq. 14 gives $E_{2}=(3050 \pm 370) \mathrm{Pa}$; $d E_{2} / E_{2}=d h / h \simeq 12 \%$ in the viscoelastic part of the deformation. We also deduce $\eta=(57 \pm 8)$ Pa.s from the fitted rising time scale $\tau_{v}=\eta / E_{2}$, which is in agreement with the value $\eta=62$ Pa.s measured for a concentration of $2.2 \% \mathrm{wt} ; 53$ for the estimation of the error, we assumed $d \eta / \eta \simeq d \tau_{v} / \tau_{v}+d E_{2} / E_{2}$ where $d \tau_{v}$ is the standard error given by the fit. Concomitantly, the fit of the amplitude of the relaxing deformation leads to $E_{2}=(3350 \pm 370) \mathrm{Pa}\left(d E_{2} / E_{2}=d h / h \simeq 11 \%\right.$ for the relaxing signal), which is $10 \%$ larger than the rising value, but compatible when considering the calculated error. 

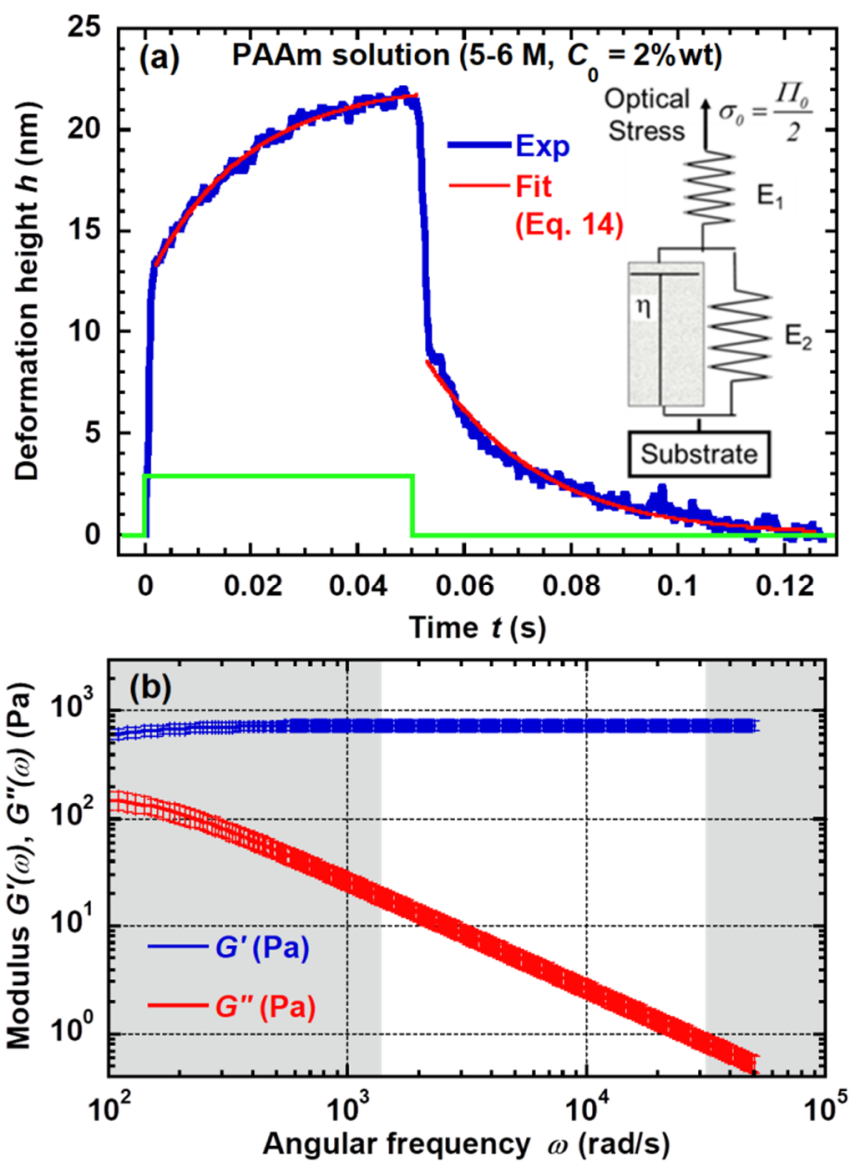

Figure 6: (a) Time-resolved interface deformation of an evaporating DI water Polyacrylamide (5-6M) drop of initial concentration $C_{0}=2 \% \mathrm{wt}$; the used power and waist are $P=3 \mathrm{~W}$ and $\omega_{0}=175 \mu \mathrm{m}$; the continuous red lines are data fits according to Eq. 14. Inset: Schematics of the one-dimension Standard Linear Solid model ${ }^{52}$ used for the data analysis and fitting (Eq. 14). (b) Storage $G^{\prime}(\omega)$ and loss $G^{\prime \prime}(\omega)$ moduli variations deduced from the mean elasticity and viscosity values obtained by fitting the interface deformation (a); the white area represents the frequency range associated to the measurements.

From the fit value of $\tau_{v}=\eta / E_{2}$, we end up with $\eta=(66 \pm 9) \mathrm{Pa} . \mathrm{s}$ which is also $10 \%$ larger than the rising value but compatible when considering the error. Finally, it could be useful to illustrate these results in the frequency domain and compare the range of application of our optical technique with those of other methods. To do so, we inject the mean measured values of $E_{1}, E_{2}$ and $\eta$ into the creep compliance $D=\varepsilon_{\uparrow}\left(t<t_{0}\right) / \sigma_{0}$ (Eq. 14), and switch to frequency domain with a s-multiplied Laplace transform (so-called Carson transform) in order to determine the two components $D^{\prime}$ and $D^{\prime \prime}$ of the complex creep compliance $D^{*}(\omega)=D^{\prime}(\omega)-i D^{\prime \prime}(\omega):{ }^{54}$

$$
\left\{\begin{array}{l}
D^{\prime}(\omega)=E_{1}^{-1}+E_{2}^{-1} /\left[1+\left(\omega \tau_{v}\right)^{2}\right], \\
D^{\prime \prime}(\omega)=\left(\omega \tau_{v}\right) E_{2}^{-1} /\left[1+\left(\omega \tau_{v}\right)^{2}\right],
\end{array}\right.
$$

where $\omega$ is the angular frequency associated to the interface deformation dynamics. The two components $E^{\prime}(\omega)$ and $E^{\prime \prime}(\omega)$ of the complex Young modulus $E^{*}(\omega)=E^{\prime}(\omega)+i E^{\prime \prime}(\omega)$ are then deduced from the relation $D^{*}(\omega) \cdot E^{*}(\omega)=1$ :
$\left\{\begin{array}{l}E^{\prime}(\omega)=D^{\prime}(\omega) /\left[D^{\prime 2}(\omega)+D^{\prime \prime 2}(\omega)\right], \\ E^{\prime \prime}(\omega)=D^{\prime \prime}(\omega) /\left[D^{\prime 2}(\omega)+D^{\prime \prime 2}(\omega)\right] .\end{array}\right.$

Eventually, we obtain the storage $G^{\prime}(\omega)$ and the loss $G^{\prime \prime}(\omega)$ moduli of the complex shear modulus $G^{*}(\omega)=E^{*}(\omega) /[2(1+v)]$ ; we assume PAAm solutions as incompressible ${ }^{47}$ so that the Poisson ratio is $v \simeq 1 / 2$. Fig. $6 b$ illustrates the expected variations of $G^{\prime}(\omega)$ and $G^{\prime \prime}(\omega)$ over the angular frequency range $1400<\omega<32000 \mathrm{rad} / \mathrm{s}$ corresponding respectively to the $50 \mathrm{~ms}$ exposure to radiation pressure and to the $0.2 \mathrm{~ms}$ temporal resolution of the acquired signal; errors bars are obtained by differentiating the expressions of $G^{\prime}(\omega)$ and $G^{\prime \prime}(\omega)$ and using the mean measured values of $d E_{1} / E_{1}, d E_{2} / E_{2}$ and $d \eta / \eta$. In addition to classical rheometers, including $A F M, 55$ it appears that interface deformation by the radiation pressure gives naturally access to material response at high frequencies where the PAAm behaviour is mainly dominated by elastic contributions; investigation of the rheology at smaller angular frequencies, in particular to get insights on the frequency-dependent viscous contribution, can be foreseen by decreasing the evaporation rate.

\section{Comment on laser heating effects}

In previous experiments, we considered optically transparent fluids that do not absorb light at the pump laser wavelength in order to demonstrate the capability of pure radiation pressure effects for the investigation of the rheological properties of fluids. However, any complex fluid, such as natural gels for instance, absorbs light almost all over the visible spectrum. So it should result some inhomogeneous heating during laser actuation, even if the latter is weak in amplitude, which may change the physical properties of the fluid under investigation and lead to complexity in signal interpretations. ${ }^{39}$ This is one of the reasons why our optical method uses interferometric detection instead of interface curvature measurement; $25,29,32-39$ by doing so, we significantly reduce the pump beam intensity and thus the energy deposited in the fluid. Consequently, laser heating can be limited and for fluids that roughly look transparent, natural ones in particular, bulk properties (density, viscosity and index of refraction) variations are likely to be negligible. This is even more true when considering the fact that our investigation is devoted to small thicknesses for which (i) it can be assumed that $\alpha_{a b s} h_{0}<<1$, where $\alpha_{a b s}$ is the optical absorption at the used wavelength, and (ii) the threshold for Rayleigh-Bénard convection, behaving as $h_{0}{ }^{3}$, is far from being reached. ${ }^{56}$ Nonetheless, if bulk heating effects can be confidently neglected for weakly absorbing fluids, radial thermal gradients $\nabla T$ induced by focused light may be very large compared to classical situations because the beam waist remains intrinsically small. Consequently, as both surface tension $\gamma(T)$ and density $\rho(T)$ depend on temperature, it results (i) a surface tension gradient that triggers a tangential thermocapillary stress along the interface directed towards 

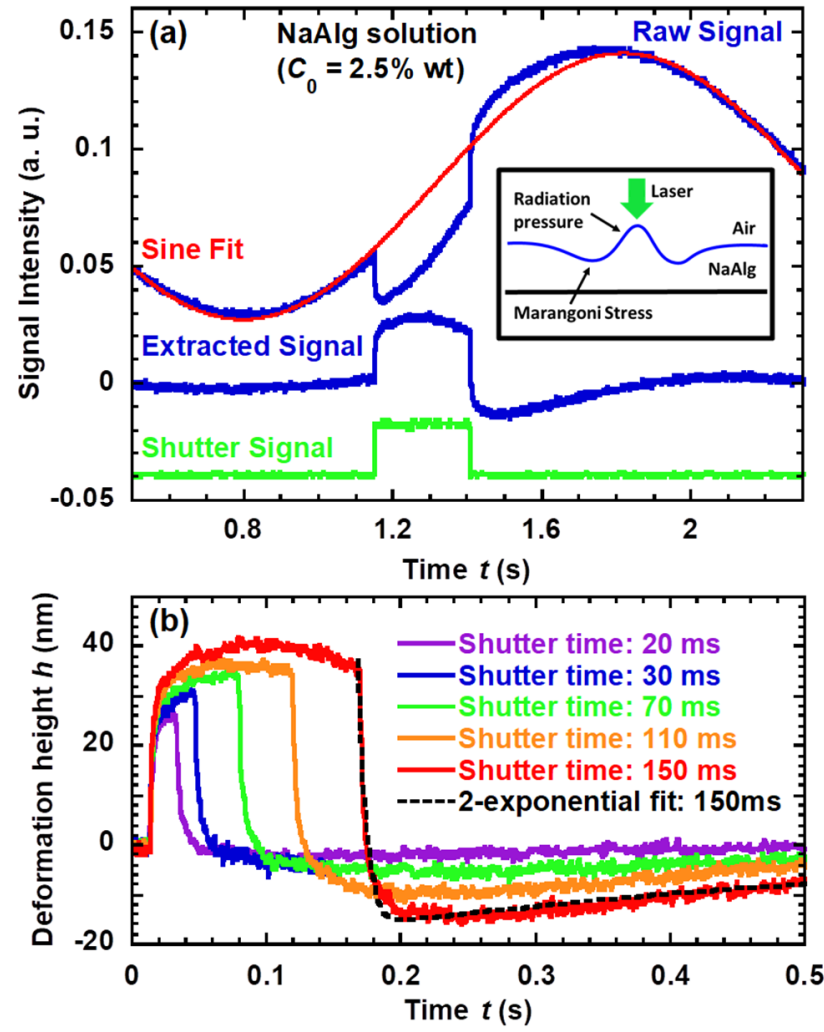

Figure 7: (a) Time-resolved interface deformation of an evaporating DI watersodium alginate ( $\mathrm{NaAlg}$ ) drop of initial concentration $C_{0}=2.5 \% \mathrm{wt}$; the used power and waist are $P=4 \mathrm{~W}$ and $\omega_{0}=105 \mu \mathrm{m}$. Two contributions appears in the interface deformation, a fast one due to radiation pressure and a slow one in opposite direction triggered by thermocapillary stresses associated to a weak optical absorption of the solution. Inset: schematics of an interface deformed by normal radiation pressure and tangential thermocapillary stresses. (b) Superimposed deformation heights obtained for increasing exposure times; the dashed line shows a two-exponential fit of the relaxation of the deformation obtained after $150 \mathrm{~ms}$ exposure.

regions of highest surface tension and (ii) a thermogravitational pressure gradient that produces a radial flow within the deposited drop. Imposing a thermal gradient $\nabla T$ and using linear developments of $\gamma(T) \approx \gamma_{0}+(\partial \gamma / \partial T)\left(T-T_{0}\right)$ and $\rho(T) \approx \rho_{0}+(\partial \rho / \partial T)\left(T-T_{0}\right)$, where $\gamma_{0}$ and $\rho_{0}$ are the surface tension and the density at the ambient temperature $T_{0}$

, Birikh ${ }^{57}$ calculated these two flows in the lubrication approximation. From mass conservation, he deduced the resulting interface deformation of a liquid layer induced by a horizontal thermal gradient:

$\nabla h_{T h}(t \rightarrow \infty)=\left[\frac{3}{2 \rho_{0} g h_{0}}\left(\frac{\partial \gamma}{\partial T}\right)-\frac{3 h_{0}}{8 \rho_{0}}\left(\frac{\partial \rho}{\partial T}\right)\right] \nabla T$.

Since $\partial \gamma / \partial T<0$ and $\partial \rho / \partial T<0$ around $T_{0}=20^{\circ} \mathrm{C}$ for classical fluids like ours, thermocapillary stresses and thermogravitational flows have opposing effects. Nonetheless, Eq. 17 shows that thermocapillarity is always dominating in our conditions since $h_{0}(\simeq 0.9 \mathrm{~mm})<<2 \sqrt{|\partial \gamma / \partial T| /(g|\partial \rho / \partial T|)}=14 \mathrm{~mm}$ for a water drop of $30 \mu \mathrm{l}$ and radius at substrate $R=4.5 \mathrm{~mm}$. Thus in presence of thermocapillarity with $\partial \gamma / \partial T<0$, the induced flow should be radially directed away from the heating laser and due to mass conservation, the resulting toroidal flow should produce a beam-centred dimple on the top of the drop interface. ${ }^{19-21}$ In the viscous regime, ${ }^{58}$ the dynamics is still governed by the capillary velocity $\gamma / \eta$ and from Eq. 17, the stationary height on beam axis of the thermocapillary deformation $h_{T h}(r=0, t \rightarrow \infty)$ in the linear regime behaves as:

$h_{T h}(r=0, t \rightarrow \infty) \simeq-\frac{3}{2 \rho g h_{0}}\left|\frac{\partial \gamma}{\partial T}\right| \Delta T(r=0)$,

where $\Delta T(r=0)$ is the pump laser overheating on beam axis. Eq. 18 shows that the deepness of this dimple is even more pronounced when decreasing the thickness $h_{0}$. Then, in presence of a weak optical absorption, such a thermocapillary interface deformation should superimpose in opposite direction to the radiation pressure one ${ }^{21}$ when performing an experiment of optorheology. This is schematically illustrated in the Inset of Fig. 7a. Fig. 7a shows as well a typical signal obtained with an evaporating sessile drop of sodium alginate (Sigma-Aldrich), NaAlg, diluted in DI water at $C_{0}=2.5 \% \mathrm{wt}$; its optical absorption at $532 \mathrm{~nm}$ measured with a UV-VIS spectrometer (Shimadzu UV3600), is $\alpha_{a b s}=0.16 \mathrm{~cm}^{-1}$. The initial drop volume is $V_{0}=30 \mu \mathrm{l}$ and its radius at contact on the prism is $R=4.5 \mathrm{~mm}$ allowing investigation of radiation pressure effects in the 'thick' layer regime under condition $\alpha_{a b s} h_{0} \ll<$; we used $P=4 \mathrm{~W}$ and $\omega_{0}=105 \mu \mathrm{m}$ for the optical actuation. As shown in Fig. 7a, the interface deformation signal starts by a fast increase, likely related to radiation pressure actuation as in previous experiments (time scale $\tau_{\text {Thick }} \simeq 2 \eta \omega_{0} / \gamma$ with optical length scale $\left.\omega_{0}\right)$; using $\gamma \simeq 0.06 \mathrm{~N} / \mathrm{m}$ and $\eta \simeq 1.5$ Pa.s for the concentration $C_{0}=2.5 \% \mathrm{wt}, 59$ the estimated value is $\tau_{\text {Thick }} \simeq 5.3 \mathrm{~ms}$. Then, the signal progressively reaches a maximum around $100 \mathrm{~ms}$ and decreases with a much larger time scale, likely related to the laser heating distribution of length scale $\omega_{\text {Th }} \gg \omega_{0}$ since heating, as any dissipative process, highly depends on boundary conditions. ${ }^{60,} 61$ Then, we expect $h_{0} / \omega_{\text {Th }}<1$ for $h_{0}=895 \mu \mathrm{m}$, which means that the dynamics of the interface deformation induced by thermocapillary actuation starts to belong to the 'thin' film regime of time scale $\tau_{\text {Th }} \simeq 3 \eta \omega_{\text {Th }}{ }^{4} / \gamma h_{0}{ }^{3}$ (Eq. 9). Fig. 7b shows this expected long-term dynamics when several deformations, acquired for decreasing exposure time, are superimposed. The opposition in direction of interface deformation between radiation pressure and thermocapillary actuation, as well as their difference in time scales, are even more noticeable when the pump laser is turned off. At first, the interface relaxes fast (according to the radiation pressure time scale $\left.\tau_{\text {Thick }} \simeq 2 \eta \omega_{0} / \gamma\right)$ to a position below the top of the non-deformed drop (negative height), demonstrating that a dimple has indeed been induced. Then this dimple relaxes much slower than expected from radiation pressure effects. Assuming a linear dynamics of relaxation with two wellseparated characteristic time scales, we fit the relaxation after $150 \mathrm{~ms}$ exposure (illustrated in Fig. 7b) considering empirically a two-exponential behaviour $h(t)=\alpha \cdot \exp \left[-\left(t-t_{0}\right) / \tau_{\text {Thick }}\right]-\beta \cdot \exp \left[-\left(t-t_{0}\right) / \tau_{T h}\right]$ with time scales $\tau_{\text {Thick }}$ and $\tau_{\text {Th }}$. We find (i) a value $\tau_{\text {Thick }}=6 \mathrm{~ms}$ very close 
to the previous estimation from the literature $\left(\tau_{\text {Thick }} \simeq 5.3 \mathrm{~ms}\right.$ ), 59 and (ii) a ratio $\tau_{\text {Th }} / \tau_{\text {Thick }}=3 / 2 \cdot \omega_{\text {Th }}{ }^{4} /\left(\omega_{0} h_{0}{ }^{3}\right)=440 / 6$. Using $h_{0}=895 \mu \mathrm{m}$ and $\omega_{0}=105 \mu \mathrm{m}$, we finally obtain $\omega_{\text {Th }} \simeq 1385 \mu \mathrm{m}$ which gives a ratio $\omega_{T h} / \omega_{0} \simeq 13$ in between those measured for laser-heated water layers of thicknesses $h_{0}=28 \mu \mathrm{m}$ $\left.\omega_{T h} / \omega_{0} \simeq 4\right)^{61} \quad$ and $h_{0}=480 \mu \mathrm{m} \quad\left(\omega_{T h} / \omega_{0} \simeq 30\right)^{62}$ when sandwiched between two glass slides; we also verify retrospectively that $\omega_{T h} / \omega_{0} \gg 1$ and $h_{0} / \omega_{T h}=0.65<1$. Interestingly, these well-separated times scales $\tau_{\text {Thick }}$ and $\tau_{\text {Th }}$ may allow a partial disentanglement of radiation pressure and thermocapillary deformations for Newtonian fluids and then, may offer the opportunity to determine some temperature dependence in fluid properties. Going farther in such a quantitative description is nonetheless far beyond the scope of the present investigation since (i) a dynamic model taking into consideration both radiation pressure and thermocapillary stresses still needs to be developed, and (ii) reliable verification of pure thermocapillary actuation requires a different pump beam wavelength, ideally in the IR for efficient heating at beam powers low enough to significantly reduce radiation pressure effects, typically in the $1440-1480 \mathrm{~nm}$ range for water and water mixtures.

\section{Conclusions}

We developed a new time-resolved optical strategy for the investigation of the rheology of viscous fluids from nanodeformations of fluid interfaces. This strategy is local, active and contactless, and allows for in situ investigations of the rheology of Newtonian and non-Newtonian fluids, from bulk to confined micrometric volumes such as sessile drops or thin soft films. Using (i) the dynamical deformation of an interface by the optical radiation pressure of a pump laser beam and (ii) the interferometric measurement of this nanometric deformation with a second probe beam, we demonstrated the capabilities of this new optical approach by measuring the surface tension and the viscosity of nonevaporating and evaporating Newtonian liquids; we showed in this last case how one can characterise in a single run the dependence of the dynamics with the film thickness, and the viscosity dependence in concentration of binary liquid mixtures and liquid suspensions. Moreover, we demonstrated the efficiency of radiation pressure for the investigation of the rheology of elastic and viscoelastic soft materials such as transparent polymer solutions. Depending on the available power of the pump beam, the range of largest measurable surface tensions is given by the condition $|d h| / h<<1$, where $h \approx \Pi_{0} \omega_{0}^{2} / \gamma$ is the order of magnitude of the light-induced interface deformation and $|d h| \approx 1 \mathrm{~nm}$ is the actual resolution of our setup. Alongside, the largest measurable viscosities are given by fulfilling the condition $h / \tau_{\text {Exp }}>>\left|d h_{0} / d t\right|$ for evaporating liquids, and $h / \tau_{E x p} \gg S$ for non-evaporating liquids, where $S$ is the slope of the saw-teeth variation of the piezo-actuation of the lens L2. This investigation centred on the viscous regime can easily be extended to any other dynamic regime from weakly damped to inertial, ${ }^{24}$ particularly when considering large layer thicknesses $h_{0}$. It can as well be developed for microdroplet rheology by taking into account in the modelling the initial interface curvature and the finite sizes in all directions. ${ }^{63}$ Eventually, we showed that our method should be extendable to thermocapillary actuation of interfaces when fluids under investigation weakly absorb light, offering a way to determine the temperature dependence of the viscosity $\eta(T)$ from the dynamics and the important interfacial quantity $\partial \gamma / \partial T$ from the amplitude of deformation.

\section{Conflicts of interest}

There is no conflict to declare.

\section{Acknowledgements}

The authors acknowledge financial support from the Agence Nationale pour la Recherche ANR (Project CAPILLARY WINDLASS No. ANR-14-CE07-0023-02). The authors thank Thomas Salez for enlightening discussions on thin film dynamics, Michele Ash and Hongyue Cui for their participation to experiments during their internships, and the Machine and Electronic shops of the LOMA for their technical assistance.

\section{References}

1 A. Erdemir, Tribology International, 2005, 38, 249.

2 S. F. Kistler and P. M. Schweizer, Liquid Film Coating: Scientific Principles and Their Technological Implications, Chapman \& Hall Editors, 1997.

3 H. Teyssèdre, S. Landis, P. Gilormini and G. Régnier, Applied Physics A, 2015, 121, 387.

4 A. Oron, S. H. Davis and S. G. Bankoff, Reviews of Modern Physics, 1997, 69, 931.

5 R. V.Craster and O. K. Matar, Reviews of modern physics, 2009, 81, 1131.

6 R. Blossey, Thin liquid films: dewetting and polymer flow, Springer Science \& Business Media, 2012.

7 A. Mathiazhagan and R. Joseph, International Journal of Chemical Engineering and Applications, 2011, 2, 225.

8 E. Rio, A. Daerr, F. Lequeux and L. Limat, Langmuir, 2006, 22, 3186.

9 M. Driver, Coatings for biomedical applications, Elsevier, 2012.

10 P. Vettiger, G. Cross, M. Despont, U. Drechsler, U. Dürig, B. Gotsmann, W. Häberle, M. A. Lantz,H. E. Rothuizen, R. Stutz and G. K. Binnig, IEEE Transactions on nanotechnology, 2002, 1, 39. 
11 Q. D. Ling, D. J. Liaw, C. Zhu, D. S. H. Chan, E. T., Kang and K. G. Neoh, Progress in polymer science, 2008, 33, 917.

12 O. Bäumchen, R. Fetzer and K. Jacobs, Physical Review Letters, 2009, 103, 247801.

13 M. Backholm, M. Benzaquen, T. Salez, E. Raphaël, E., and K. Dalnoki-Veress, Soft Matter, 2014, 10, 2550.

14 B. Pottier, C. Frétigny and L. Talini, Physical Review Letters, 2015, 114, 227801.

15 L. E. Stillwagon and R. G. Larson, Physics of Fluids A,1990, 2, 1937.

16 K. Sakai and Y. Yamamoto, Applied Physics Letters, 2006, 89, 211911.

17 S. Å. Ellingsen and I. Brevik, Annals of Physics, 2012, 327, 2899.

18 C. W. J. Berendsen, C. J. Kuijpers, J. C. H. Zeegers and A. A. Darhuber, Soft Matter, 2013, 9, 4900.

19 H. M. J. M. Wedershoven, C. W. J. Berendsen, J. C. H. Zeegers and A. A. Darhuber, Applied physics letters, 2014, 104, 054101.

20 S. Rubin, B. Hong and Y. Fainman, Light: Science \& Applications, $2019,8,1$.

21 K. Chaudhary and K. P. Singh, Applied Physics Letters, 2019, 115, 251103.

22 C. Cinbis and B. T Khuri-Yakub, Review of Scientific Instruments, 1992, 63, 2048

23 P. L. Marston and D. B. Thiessen, Annals of the New York Academy of Sciences, 2004, 1027, 414.

24 B. Issenmann, R. Wunenburger, H. Chraibi, M. Gandil and J. P. Delville, Journal of Fluid Mechanics, 2011, 682, 460.

25 K. Sakai, D. Mizumo and K. Takagi, Physical Review E, 2001, 63 043602.

26 R. Wunenburger, A. Casner and J. P. Delville, Physical Review E, 2006, 73, 036314; 2006, 73, 036315.

27 A. Ashkin and J. M. Dziedzic, Physical Review Letters, 1973, 30, 139.

28 A. Casner and J. P. Delville, Physical Review Letters, 2001, 87, 054503.

29 N. G. Astrath, L. C. Malacarne, M. L. Baesso, G. V. Lukasievicz and S. E. Bialkowski, Nature Communications, 2014, 5, 4363.

30 G. Verma and K. P. Singh, Physical Review Letters, 2015, 115, 143902.

31 A. Casner and J. P. Delville, Optics letters, 2001, 26, 1418.

32 D. C. Clark and M. K. Kim, Optics letters, 2012, 37, 5145.

33 O. A. Capeloto V. S. Zanuto, L. C. Malacarne, M. L. Baesso, G. V. B. Lukasievicz, S. E. Bialkowski and N. G. C. Astrath, Scientific reports, 2016, 6, 20515.

34 S. Mitani and K. Sakai, Physical Review E, 2002, 66, 031604.

35 T. Takei, T. Yaguchi, T. Fujii, T. Nomoto, T. Toyota and M. Fujinami, Soft Matter, 2015, 11, 8641.

36 Y. Yoshitake, S. Mitani, K. Sakai and K. Takagi, Journal of Applied Physics, 2005, 97, 024901.
37 F. Wottawah, S. Schinkinger, B. Lincoln, R. Ananthakrishnan, M. Romeyke, J. Guck and J. Käs, Physical Review Letters, 2005, 94, 098103.

38 T. Morisaku and H. Yui, Analyst, 2018, 143, 2397.

39 Y. Yoshitake, S. Mitani, K. Sakai, and K. Takagi, Physical Review $E, 2008,78,041405$.

40 M. Benzaquen, M. Ilton, M. V. Massa, T. Salez, P. Fowler, E. Raphaël and K. Dalnoki-Veress, Applied Physics Letters, 2015, 107, 053103.

41 G. Verma and K. P. Singh, Applied Physics Letters, 2014, 104, 244106.

42 C. Illueca, C. Vazquez, C. Hernandez, V. Viqueira, Ophthalmic and Physiological Optics, 1998, 18, 360.

43 G. V. Ostrovskaya, Soviet Physics and Technical Physics, 1988 33, 465 ; I. I. Komissarova, G. V. Ostrovskaya and E. N. Shedova, Soviet Physics and Technical Physics, 1988, 33, 468.

44 M. L. Henle and A. J. Levine, Physical Review E, 2007, 75, 021604.

45 W. M. Haynes, CRC handbook of chemistry and physics: A readyreference book of chemical and physical data. Boca Raton CRC Press (2009).

46 C. Poulard, G. Guéna, A. M. Cazabat, A. Boudaoud and M. Ben Amar, Langmuir, 2005, 21, 8226.

$47 \mathrm{H}$. Chraibi, D. Lasseux, E. Arquis, R. Wunenburger and J. P. Delville, European Journal of Mechanics-B/Fluids, 2008, 27, 419.

48 A. Volk and C. J. Kähler, Experiments in Fluids, 2018, 59, 75.

49 K. Takamura, H. Fischer and N. R. Morrow, Journal of Petroleum Science and Engineering, 2012, 98, 50

50 G. D'Errico, O. Ortona, F. Capuano and V. Vitagliano, Journal of Chemical \& Engineering Data, 2004, 49, 1665.

51 J. B. Segur and H. E. Oberstar, Industrial and Engineering Chemistry, 1951, 43, 2117.

52 N. W. Tschoegl, The Phenomenological Theory of Linear Viscoelastic Behavior. Springer-Verlag Berlin Heidelberg (1989).

53 W. M. Kulicke, R. Kniewske and J. Klein, Progress in Polymer Science, 1982, 8, 373

54 S. W. Park and R. Schapery, International journal of solids and structures, 1999, 36, 1653.

55 Y. Abidine, V. M. Laurent, R. Michel, A. Duperray, L. I. Palade and C. Verdier, Europhysics Letters, 2015, 109, 38003.

56 D. Gutkowicz-Krusin, M. A. Collins and J. Ross, Physics of Fluids, 1979, 22, 1451.

57 R. V. Birikh, Journal of Applied Mechanics and Technical Physics, 1966, 7, 43.

58 H. Chraibi and J. P. Delville, Physics of Fluids, 2012, 24, 032102.

59 P. Del Gaudio, P. Colombo, G. Colombo, P. Russo and F. Sonvico, International Journal of Pharmaceutics, 2005, 302, 1.

60 J. P. Gordon, R. C. C. Leite, R. Moore, S. P. S. Porto and J. R. Whinnery, Journal of Applied Physics, 1965, 36, 3. 
61 M. L. Cordero, E. Verneuil, F. Gallaire and C. N. Baroud, Physical Review E, 2009, 79, 011201.

62 D. Rivière, B. Selva, H. Chraibi, U. Delabre and J. P. Delville, Physical Review E, 2016, 93, 023112.

63 H. Chraibi, D. Lasseux, E. Arquis, R. Wunenburger and J. P. Delville, Physical Review E, 2008, 77, 066706. 